\title{
Measurement considerations on examiner-dependent factors in the ultrasound assessment of developmental dysplasia of the hip
}

\author{
Alexander Kolb $^{1}$ (D) $\cdot$ Emir Benca $^{1} \cdot$ Madeleine Willegger $^{1}$ • Stephan E. Puchner ${ }^{1}$ • \\ Reinhard Windhager $^{1} \cdot$ Catharina Chiari $^{1}$
}

Received: 20 September 2016 / Accepted: 20 March 2017 /Published online: 12 April 2017

(C) The Author(s) 2017. This article is an open access publication

\begin{abstract}
Purpose The standardized sonographic hip screening according to Graf has increased reliability and comparability of measurements in the screening of developmental dysplasia of the hip (DDH). However, examiner dependent factors have been discussed to influence sonographic measurements. The objectives of this study were to examine the tolerance of the transducer positioning and to analyse the impact of transducer inclinations on Graf's hip grading system.

Materials and methods Twenty-four hips in consecutive newborns were screened sonographically in combination with an optoelectronic motion capture system to trace transducer positions in space. Subsequently five defined inclinations of the
\end{abstract}

Electronic supplementary material The online version of this article (doi:10.1007/s00264-017-3455-9) contains supplementary material, which is available to authorized users.

Alexander Kolb

alexander.kolb@meduniwien.ac.at

Emir Benca

emir.benca@meduniwien.ac.at

Madeleine Willegger

madeleine.willegger@meduniwien.ac.at

Stephan E. Puchner

stephan.puchner@meduniwien.ac.at

Reinhard Windhager

reinhard.windhager@meduniwien.ac.at

Catharina Chiari

catharina.chiari@meduniwien.ac.at

1 Department of Orthopaedics and Orthopaedic Surgery, Medical University of Vienna, Waehringer Gürtel 18-20,

1090 Vienna, Austria transducer relative to Graf's neutral transducer position were analysed, giving a total of 144 sonographic images.

Results We found a permissible transducer inclination in the axial plane of $8.8^{\circ}$ to anterior and $8.1^{\circ}$ to posterior. In the frontal plane we found a permissible inclination of $15.4^{\circ}$ to caudal and $7.2^{\circ}$ to cranial. The impact on the $\alpha$-angle was significant for posterior-cranial $(p<0.001)$, cranial $(p=0.009)$, and caudal $(p<0.001)$ inclined transducer positions. The effect on the results according to Graf's grading system was significant for the caudal inclination of the transducer position $(p<0.001)$.

Conclusion Our findings show that the standardized plane defined by Graf's criteria allows notable inclinations of the transducer positions. Transducer inclinations show an impact on measurement results, which are clinically relevant. Those effects cannot be ruled out using Graf's ultrasound criteria alone. The examiner should pay attention to avoid transducer inclinations in the frontal plane and a combination of posterior and cranial inclination.

Keywords Hip sonography · Developmental Dysplasia of the Hip (DDH)

\section{Introduction}

A relatively wide range of incidence rates of developmental dysplasia of the hip (DDH) is reported among the literature. Besides cohort dependent factors different definitions and screening methods for DDH are known issues in this context. Thus, a standardized sonographic hip screening method like the sonographic method according to Graf et al. is expected to increase the reliability and comparability of given incidence rates [1]. 
Several studies were conducted and proved Graf's broadly used sonographic screening method for DDH to be reliable and reproducible [2-4]. However, previous studies focused on the method of measurement on given sonographic images, whereas the process of acquisition of sonographic images was not examined. Considering the clinical importance of Graf's method in established national screening programs for DDH in many European countries, it is interesting that an essential issue like the link of transducer inclinations to measurement results was not examined before $[5,6]$.

The screening method according to Graf is based on a defined, standardized transducer position $[7,8]$. The transducer position is determined by the appearance of anatomical structures (landmarks) and their defined cross-sectional shape on the sonographic images. Graf described the standardized transducer positon as a position within an ideal plane defined by his criteria. However, in reality the criteria define a set of possible transducer positions including inclinations in the axial and frontal plane. Interestingly there is only little evidence on the tolerance of transducer positions and the resulting effect on measurement results [9]. Despite the lack of evidence on the tolerance of transducer positions and its effect on measurement results, the problem has been reported and technical improvements ("Sono-Guide") were suggested in order to limit this cause of examiner dependent measurement deviations [10]. However, despite the use of this standardized method a relatively wide range $(0.24 \%$ to $4.7 \%)$ of incidence rates of DDH were reported [11-15].

Thus, one question remains: How big is the examiner dependent influence in the process of acquisition of sonographic images?

The objectives of this study were (1) to examine the permissible tolerance of the transducer positioning and (2) to analyse the resulting effect on the hip measurement and grading system.

\section{Materials and methods}

Institutional review board approval was obtained for this study at our institution. All patients or legal guardians gave their informed consent prior to the inclusion in this study.

Twelve consecutive newborns (24 hips) delivered at one hospital were included in the study. A feed-and-sleep technique was used to put the newborns to sleep prior to the measurement. The sonographic screening process was video monitored. The examination was interrupted when the newborns started to move.

The newborns were screened using Graf's ultrasound method within the first neonatal week. Ultrasound screening was performed using a GE logiq 200 pro series system and a 7.5-MHz LH linear transducer (GE Healthcare, Milwaukee, WI, USA). The newborns were examined in lateral position using a positioning device (Sono-Fix, Gebrüder Hirschbeck
$\mathrm{GmbH}$, Austria) as recommended by Graf [9]. All newborns underwent a standardized physical examination including the Barlow and Ortolani tests [16, 17], test of hip abduction, assessment of femoral length discrepancies, asymmetries and associated deformities.

Three-dimensional positions in space of the transducer during the whole ultrasound screening process were recorded using an optoelectronic motion capture system Smart-E (BTS Bioengineering, Milan, Italy). The motion capture system is based on optical technology using four infrared cameras sampling at $120 \mathrm{~Hz}$. Passive infrared markers were rigidly attached to the transducer. A three-dimensional reconstruction of trajectories of the markers was preformed using the Smartanalyzer software (BTS Bioengineering, Milan, Italy). Positions in space of the transducer synchronized to points in time of the sonographic image acquisitions were extracted.

In each new-born, sonographic images of the hip were obtained in the following order starting with a standardized position according to Graf which felt most neutral to the examiner. In this neutral position the examiner was encouraged to avoid any specific inclination of the transducer. Subsequently sonographic measurements in a position with a tendency of inclination to anterior, posterior, cranial and caudal were obtained. The amount of inclination in these positions was limited with respect to the criteria described by Graf. Finally, a transducer position using a combination of posterior and cranial inclination was examined (Fig. 1 and Fig. 2).

The analysis of the sonographic images was performed blinded to patient and transducer position data. All sonographic images were checked according to Graf's criteria prior to further analysis and only appropriate sonographic images were processed. Sonographic measurements of alpha- and beta-angels according to Graf and matched transducer position in space were

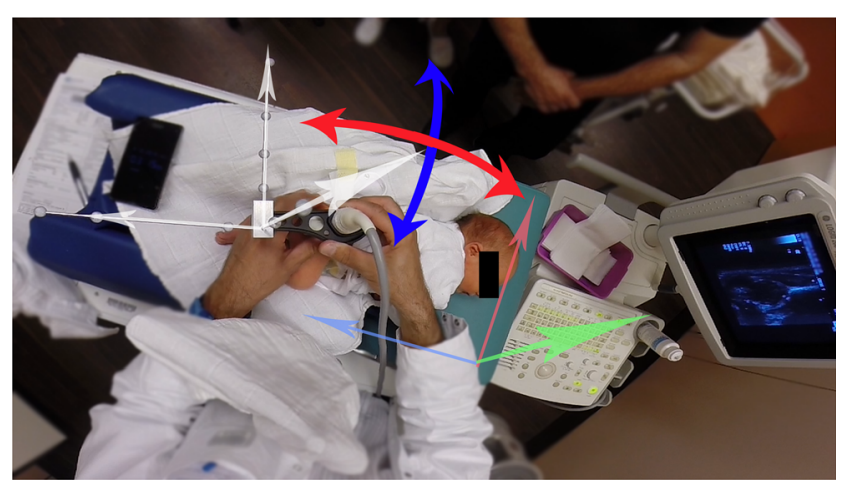

Fig. 1 Photograph of the measurement setup, showing a newborn lying in the positioning device and the passive infrared markers rigidly attached to the transducer. Unit vectors of the transducer position are represented by three white arrows, unit vectors of the coordinate system relative to the positioning device are represented by the blue, red and green arrows. Transducer inclination to anterior and posterior (blue double arrow) and transducer inclination to cranial and caudal (red double arrow) were measured as rotation around the blue and red unit vector relative to the neutral transducer position 


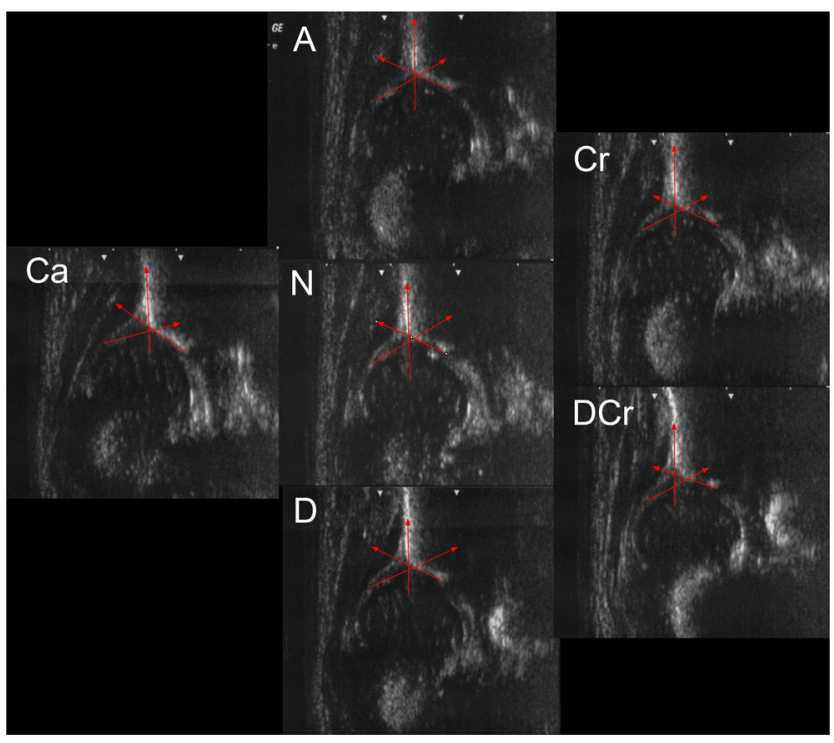

Fig. 2 Series of sonographic images of the same physiological hip joint (Graf type Ib) acquired in different transducer positions and measurement lines according to Graf (red lines) showing an $\alpha$-angle range of $54^{\circ}$ to $71^{\circ}$. Neutral position (N) $\alpha=64, \beta=60$; anterior position (A) $\alpha=65$, $\beta=60$; dorsal position (D) $\alpha=64, \beta=65$; caudal position (Ca) $\alpha=54$, $\beta=76$; cranial position $(\mathrm{Cr}) \alpha=65, \beta=62$; and dorsocranial position (DCr) $\alpha=71, \beta=64$

analysed. Based on the defined transducer positions differences of transducer positions in space, and alpha- and beta-angles to the neutral position were calculated.

\section{Statistical analysis}

Data were processed using the SPSS 20 software (SPSS Inc., Chicago, IL, USA). Considering the coarsening nature of classification systems $\alpha$ - and $\beta$-angels were first analysed directly [18]. Differences between sonographic $\alpha$ - and $\beta$-angels on different transducer positions were analysed using two-sided paired t-tests $(p<0.05)$. The homogeneity of variances was analysed using the Levene-test. Secondly, the ordinal results based on Graf's grading system in different transducer positions compared to the neutral position were analysed using the Wilcoxon signed-rank test. The level of significance was defined as $p<0.05$.

\section{Results}

The feed-and-sleep technique was sufficient in ten of the 12 newborns. In seven of the ten newborns both hips were measured according to the study protocol, in three newborns only one hip was measured due to movement of the newborn. Primary sonographic results according to Graf's technique were type Ia in $3(17.6 \%)$, Ib in $11(64.7 \%)$ and IIa in 3 (17.6\%) hips. The results of the physical examination were normal in all newborns.
Three-dimensional (3D) measurement of defined transducer positions was performed in all measured hips: The inclination-angles of transducer positions in space relative to the neutral transducer position for anterior, posterior, cranial and caudal inclination are shown in Fig. 3. The fifth transducer position representing a combination of posterior and cranial inclination showed a mean posterior inclination of $4.2^{\circ}$ (range $0.1^{\circ}$ to $8.7^{\circ}$ ) and a mean cranial inclination of $10.5^{\circ}$ (range $5.1^{\circ}$ to $16.5^{\circ}$ ). In total we found a standard deviation of $6.6^{\circ}$ for the $\alpha$-angle, and a standard deviation of $6.6^{\circ}$ for the $\beta$ angle.

The impact of the defined transducer positions on the sonographic measurement of $\alpha$ - and $\beta$-angels relative to the sonographic measurements in neutral transducer position is given in Figs. 4 and 5. Statistical analysis revealed a significant difference of the measured $\alpha$-angles in posterior-cranial, cranial and caudal transducer positions to $\alpha$-angles measured in neutral position $\left(\mathrm{t}_{16}=7.60, p<0.001 ; \mathrm{t}_{15}=3.01, p=0.009\right.$; $\left.\mathrm{t}_{16}=-7.00, p<0.001\right)$. Differences of $\alpha$-angles in anterior and posterior inclined transducer positions to $\alpha$-angles measured in neutral position were not significant $\left(\mathrm{t}_{16}=0.11, p=0.912\right.$; $\left.\mathrm{t}_{16}=0.87, p=0.397\right)$.

Data on the frequency of hip types according to Graf seen in the defined transducer positions are given in Fig. 5. Compared to the neutral position type IIa was more frequent in anterior and posterior inclined transducer positions, and less frequent in cranial inclined transducer positions. These differences were not statistically significant $(p=0.32, p=0.66$ and $p=0.56$ ). Compared to the neutral position sonographic measurements in caudal inclined transducer positions substantially increased the number of IIa hips, and moreover type IIc and D were seen in this position. This difference was statistically significant $(p<0.001)$. All hips were classified as Graf type Ia or Ib using a combination of posterior and cranial inclination for the transducer position. However, compared to the neutral position this difference was not significant $(p=0.083)$.

\section{Discussion}

It is an interesting fact that incidence rates of DDH based on sonographic screening programs show a relatively wide range of variation $(0.24$ to $2.5 \%)$ [11, 13, 14]. Cohort dependent factors were discussed as a cause of varying incidence rates, but on the other hand examiner dependent factors have also been discussed. The standardization of the sonographic hip screening of DDH according to Graf is an important achievement improving reliability and reproducibility in this field [8]. The achievement of Graf was first to rule out, or minimize examiner dependent factors using a standardized transducer position, but there is only weak evidence on the reliability of the criteria defining the standardized transducer position. Considering the clinical importance of Graf's method in 
Fig. 3 Inclination-angles in degrees of transducer position in space relative to the neutral position to anterior, posterior, cranial and caudal

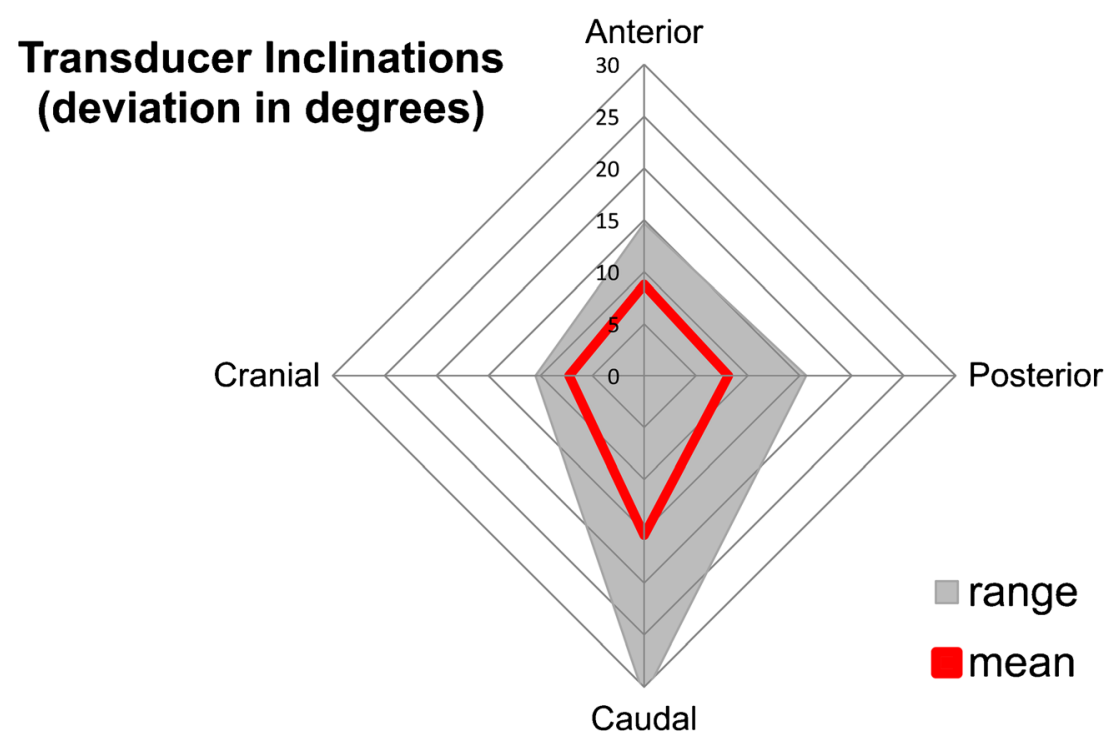

permissible cranial inclination of $7.2^{\circ}$. When testing a combination of posterior and cranial inclination for the transducer position we found a permissible combination of $4.2^{\circ}$ of posterior inclination and $10.5^{\circ}$ of cranial inclination.

The effect of transducer inclination in the frontal plane is significant for both directions: a caudal inclined transducer position significantly reduces the measured $\alpha$-angles, whereas a cranial inclined position significantly increases the measured $\alpha$-angles. A combination of posterior and cranial inclination leads to significantly increased $\alpha$-angles. Finally, the effect of different transducer positions on the sonographic measurement of $\alpha$-angles according to Graf showed a nonsignificant impact considering anterior and posterior inclined transducer positions.

The standard deviation of $\alpha$ - and $\beta$-angle found in this study is higher than reported previously. In comparison Roovers et al., who examined the reproducibility of the analysis of sonographic images, found a standard deviation of $3.2^{\circ}$ for the $\alpha$-angle, and a standard deviation of $6.0^{\circ}$ for the $\beta$ angle [20]. This supports the assumption that measuring
Fig. 4 Impact of inclined transducer positions on sonographic measurement of $\alpha$ angles

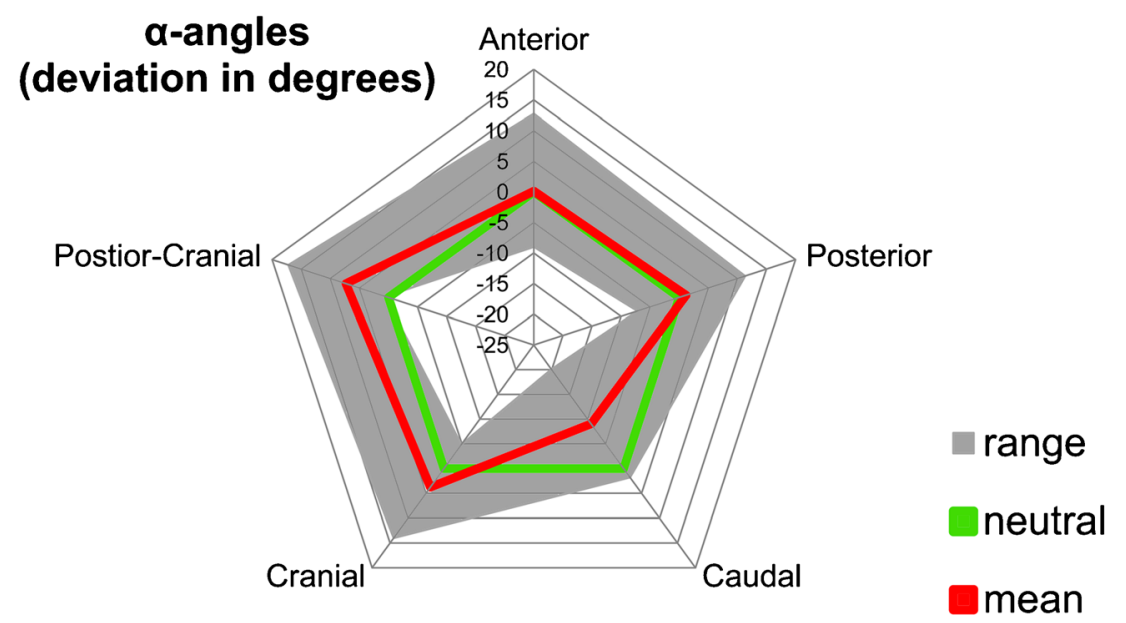


Fig. 5 Frequency (in percent) of the observed sonographic hip types according to Graf (Ia, Ib, IIa, IIc and D) in neutral (highlighted by the red box) and inclined transducer positions

\section{Impact of transducer inclination on hip classification}

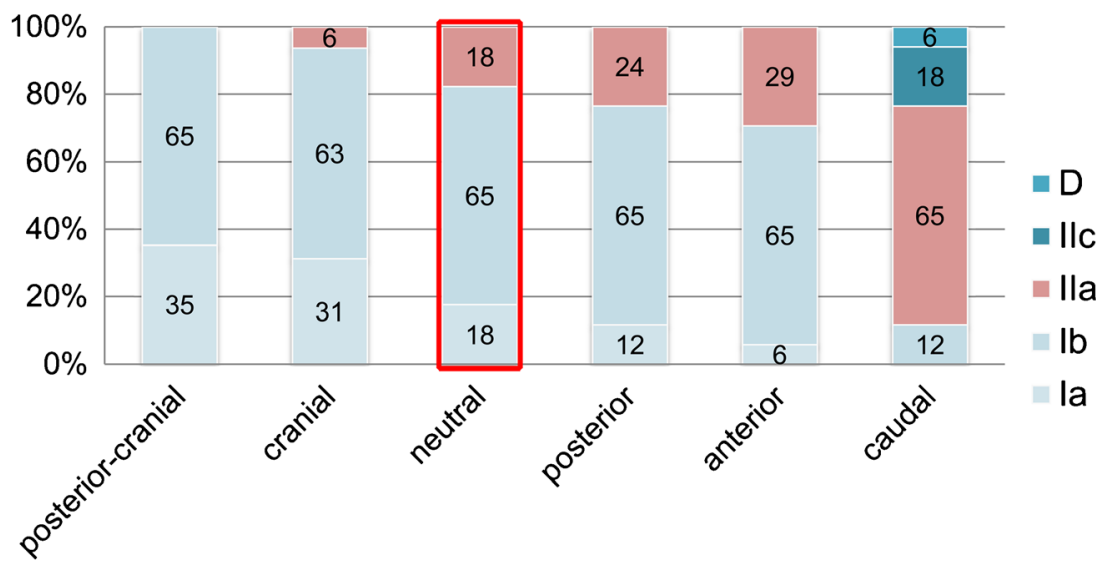

inaccuracies of the $\alpha$-angle, which is the central parameter for Graf's classification, mainly originate from the image acquisition process. However, we have to consider that measurement deviations as described by Roovers will add up to our results in the second step of analysis of the sonographic images.

The final effect of different transducer inclinations on the results classified according Graf's system is highly significant for the caudal position. Here, former normal hips were classified as type IIc and D. Therefore, this effect has potential to contribute to higher rates of reported DDH $[13,14]$.

On the contrary, this effect was reversed using a combination of posterior and cranial inclination of the transducer. In this transducer position all examined hips were classified as type Ia or type Ib, and no hips classified as type IIa anymore. An isolated cranial transducer inclination also shows a limited effect with slightly more hips classified as type Ia and type $\mathrm{Ib}$ comprared to the neutral position. Despite significant effects of these transducer positions on the $\alpha$-angels, the effect on the results according to Graf was not significant. This difference may be explained by the coarsening nature of the classification system. However, these effects may decrease the frequency of DDH especially in the first neonatal weeks in some series [11]. Considering transducer inclinations to anterior or posterior, the effect on the classification system is minimal (see Fig. 5).

In this study, the sonographic measurements showed physiological results in all included subjects. Therefore, the meaning of our results in pathologic conditions cannot be predicted directly. Based on the clinical experience of the investigators, we believe that the definition of the standard plane is less easily applicable in pathologic conditions, like Graf's type IIc, D. As a consequence, the placement of the standard plane might be less reproducible in these pathologic conditions, likely resulting in an increased variance of the sonographic $\alpha$ - and $\beta$-angles. Accordingly, this may lead to uncertainty in the classification of these hips requiring well-skilled examiners to provide a counterbalance. Some authors addressed this issue indirectly by repeating the sonographic examination in cases of mild dysplasia [21]. In this context, we have to reconsider that Graf emphasized the importance of adequate training of sonographic examiners [10].

\section{Limitations}

There are several limitations to this study. First, to analyse the relative movement between the examined hip and the transducer, we assumed that there is no movement between the sleeping newborns and the positioning device. Thus, the three-dimensional analysis recorded the transducer position relative to the positioning device. Due to technical aspects movements of the newborns were not traced directly by the optoelectronic motion capture system. The unchanged position of the newborns was monitored by video camera. Newborns, which started to move during the examination where excluded, but still minimal movements in sleeping newborns could influence the measurements.

The second important limitation is that the examiner has to decide on the possible inclination of the transducer position. The method of checking sonographic images according to Graf's criteria is sufficient to exclude excessive transducer inclinations, but it is possible that the examiner underestimates the range of allowed inclinations in this setting.

\section{Conclusion}

Our findings show that the standardized plane defined by Graf's criteria allows notable inclinations of the transducer positions. Inclinations of the transducer position in the axial plane to anterior or posterior are less critical than inclinations in the frontal plane to caudal or cranial. Most important, a 
caudal inclination leads to underestimated $\alpha$-angles, and in contrast a combined posterior and cranial transducer inclination may lead to overestimated $\alpha$-angles with subsequent effects on the classification system. The use of a mechanical device like the "Sono-Guide" might provide additional technical support. Further research is needed to define criteria and factors to optimize the transducer positioning or to minimize the impact of transducer inclinations on sonographic measurements in screening for DDH.

Acknowledgements We want to thank Mr. Manfred Franke for his support during the initial phase of this study. Open access funding provided by Medical University of Vienna.

\section{Compliance with ethical standards}

Competing interest On behalf of all authors, the corresponding author states that there is no conflict of interest. One of the authors (RW) has received funding from Boehringer Ingelheim, Pfizer, Stryker, DePuy, Takeda.

Funding This research was supported by a grant funded of the "Bürgermeisterfond der Stadt Wien".

Open Access This article is distributed under the terms of the Creative Commons Attribution 4.0 International License (http:// creativecommons.org/licenses/by/4.0/), which permits unrestricted use, distribution, and reproduction in any medium, provided you give appropriate credit to the original author(s) and the source, provide a link to the Creative Commons license, and indicate if changes were made.

\section{References}

1. Graf R (1983) New possibilities for the diagnosis of congenital hip joint dislocation by ultrasonography. J Pediatr Orthop 3:354-359

2. Roposch A, Graf R, Wright JG (2006) Determining the reliability of the Graf classification for hip dysplasia. Clin Orthop Relat Res 447: 119-124. doi:10.1097/01.blo.0000203475.73678.be

3. Rosendahl K, Toma P (2007) Ultrasound in the diagnosis of developmental dysplasia of the hip in newborns. The European approach. A review of methods, accuracy and clinical validity. Eur Radiol 17:1960-1967. doi:10.1007/s00330-006-0557-y

4. Simon EA, Saur F, Buerge M et al (2004) Inter-observer agreement of ultrasonographic measurement of alpha and beta angles and the final type classification based on the Graf method. Swiss Med Wkly 134:671-677
5. Sewell MD, Eastwood DM (2011) Screening and treatment in developmental dysplasia of the hip-where do we go from here? Int Orthop 35:1359-1367. doi:10.1007/s00264-011-1257-z

6. Sibiński M, Adamczyk E, Higgs ZCJ, Synder M (2012) Hip joint development in children with type IIb developmental dysplasia. Int Orthop 36:1243-1246. doi:10.1007/s00264-011-1447-8

7. Graf R (1980) The diagnosis of congenital hip-joint dislocation by the ultrasonic Combound treatment. Arch Orthop Trauma Surg 97: 117-133. doi:10.1097/01241398-198101010-00027

8. Graf R (1984) Fundamentals of sonographic diagnosis of infant hip dysplasia. J Pediatr Orthop 4:735-740. doi:10.1097/01241398198411000-00015

9. Graf R (1997) Hip ultrasonography. Basic principles and current aspects. Orthopade 26:14-24

10. Graf R, Mohajer M, Plattner F (2013) Hip sonography update. Quality-management, catastrophes - tips and tricks. Med Ultrason 15:299-303

11. Kolb A, Schweiger N, Mailath-Pokorny M et al (2015) Low incidence of early developmental dysplasia of the hip in universal ultrasonographic screening of newborns: analysis and evaluation of risk factors. Int Orthop. doi:10.1007/s00264-015-2799-2

12. Lehmann HP, Hinton R, Morello P, Santoli J (2000) Developmental dysplasia of the hip practice guideline: technical report. Committee on quality improvement, and subcommittee on developmental dysplasia of the hip. Pediatrics 105:E57

13. Dorn U, Neumann D (2005) Ultrasound for screening developmental dysplasia of the hip: a European perspective. Curr Opin Pediatr $17: 30-33$

14. Tönnis D, Storch K, Ulbrich H (1990) Results of newborn screening for $\mathrm{CDH}$ with and without sonography and correlation of risk factors. J Pediatr Orthop 10:145-152

15. Dogruel H, Atalar H, Yavuz OY, Sayli U (2008) Clinical examination versus ultrasonography in detecting developmental dysplasia of the hip. Int Orthop 32:415-419. doi:10.1007/s00264-007-0333-x

16. Ortolani M (1976) Congenital hip dysplasia in the light of early and very early diagnosis. Clin Orthop Relat Res 119:6-10

17. Barlow M (1937) Un segno poco noto e sua importanza per la diagnosi precoce di prelussazione congenita dell'anca. Pediatria (Santiago) 45:129-136

18. Graf R (1997) Ultrasonography-guided therapy. Orthopade 26:33-42

19. Gunay C, Atalar H, Dogruel H et al (2009) Correlation of femoral head coverage and Graf alpha angle in infants being screened for developmental dysplasia of the hip. Int Orthop 33:761-764. doi:10. 1007/s00264-008-0570-7

20. Roovers EA, Boere-Boonekamp MM, Geertsma TSA et al (2003) Ultrasonographic screening for developmental dysplasia of the hip in infants. Reproducibility of assessments made by radiographers. J Bone Joint Surg Br 85:726-730. doi:10.1302/0301-620X.85B5. 13893

21. Rosendahl K, Dezateux C, Fosse KR et al (2010) Immediate treatment versus Sonographic surveillance for mild hip dysplasia in newborns. Pediatrics 125:e9-e16. doi:10.1542/peds.2009-0357 